\title{
LOW VOLTAGE MEMS ANALOG MICROMIRROR ARRAYS WITH HIDDEN VERTICAL COMB-DRIVE ACTUATORS
}

\author{
Dooyoung Hah, Sophia Huang, Hung Nguyen, Hsin Chang, Jui-Che Tsai, and Ming C. Wu \\ Department of Electrical Engineering, University of California, Los Angeles
}

Los Angeles, CA 90095-1594

\author{
Hiroshi Toshiyoshi \\ Institute of Industrial Science, University of Tokyo \\ Tokyo, Japan 153-8505
}

\begin{abstract}
We report on a novel, polysilicon surface-micromachined one-dimensional (1-D) analog micromirror array. Large continuous DC scan angle $\left(23.6^{\circ}\right.$ optical) and low operating voltage (6V) have been achieved using vertical comb-drive actuators. The actuators and torsion springs are placed underneath the mirror $(137 \times 120$ $\mu \mathrm{m}^{2}$ ) to achieve high fill-factor $(91 \%)$. The measured resonant frequency of the mirror ranges from $3.4 \mathrm{kHz}$ to $8.1 \mathrm{kHz}$. The static scanning characteristics show good uniformity $(< \pm 3.2 \%)$ for a $1 \times$ 10 array with a mirror pitch of $150 \mu \mathrm{m}$. The measured DC scanning characteristics and resonant frequencies agree well with theoretical values. This micromirror array has applications in optical wavelength-division multiplexed (WDM) routers and wavelength-selective crossconnect (WSXC).
\end{abstract}

\section{INTRODUCTION}

The advance of photonic communication networks towards optical layer networking has created a great demand for many new functional optical network elements. Microelectromechanicalsystems (MEMS) is a key enabling technology for many of these new devices. Optical MEMS devices for dynamic optical add-drop multiplexers (OADM) [1], 2-D [2-3] and 3-D optical crossconnects $(\mathrm{OXC})$ [4] have been reported. Previously, we have reported a novel wavelength-division-multiplexed (WDM) router that employs a 1-D array of analog micromirrors [5]. Large continuous scan range and high fill factor are required to achieve high channel count and flat spectral response. In addition, low voltage, low power actuation is desired to reduce power consumption of drive electronics.

Most of the micromirrors reported to date employ parallelplate type electrostatic actuators. The pull-in phenomena in such actuators, however, limit the useful range of continuous scanning. The vertical comb-drive actuator reported recently [6, 7] offers several inherent advantages for actuating micromirrors. It can be designed to avoid pull-in effect and utilize the entire range of rotation. The large force density of vertical comb drives also lead to low voltage operation. However, the bulk-micromachined structures are not suitable for implementing high fill factor micromirror arrays where actuators are hidden underneath the mirrors. The surface-micromachining technique offers more flexibility for designing such multi-layer structures.

In this paper, we report on a novel surface-micromachined analog micromirror array with hidden vertical comb drive actuators. High fill factor $(91 \%)$, flat mirror surface $\left(137 \times 120 \mu \mathrm{m}^{2}\right)$, low operating voltage $(6 \mathrm{~V})$, and large continuous scan angle $\left(23.6^{\circ}\right.$ optical) are successfully achieved. This device is made possible by exploiting the chemical-mechanical planarization (CMP) processes in SUMMiT-V offered by Sandia National Laboratory.

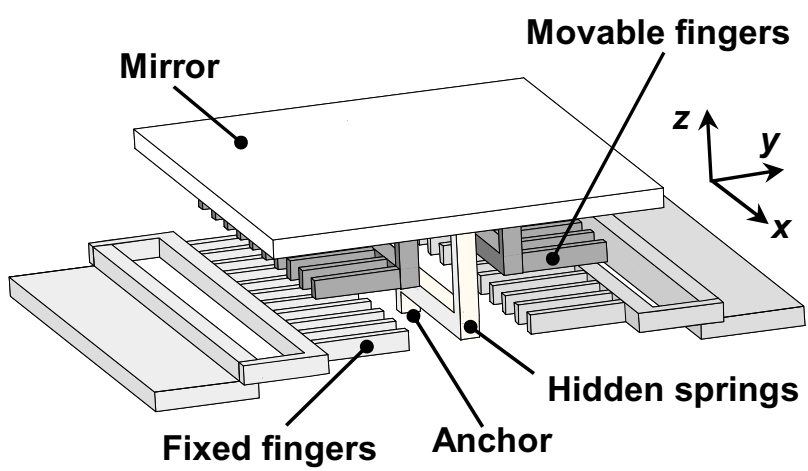

Figure 1. The schematic structure of the proposed micromirror.

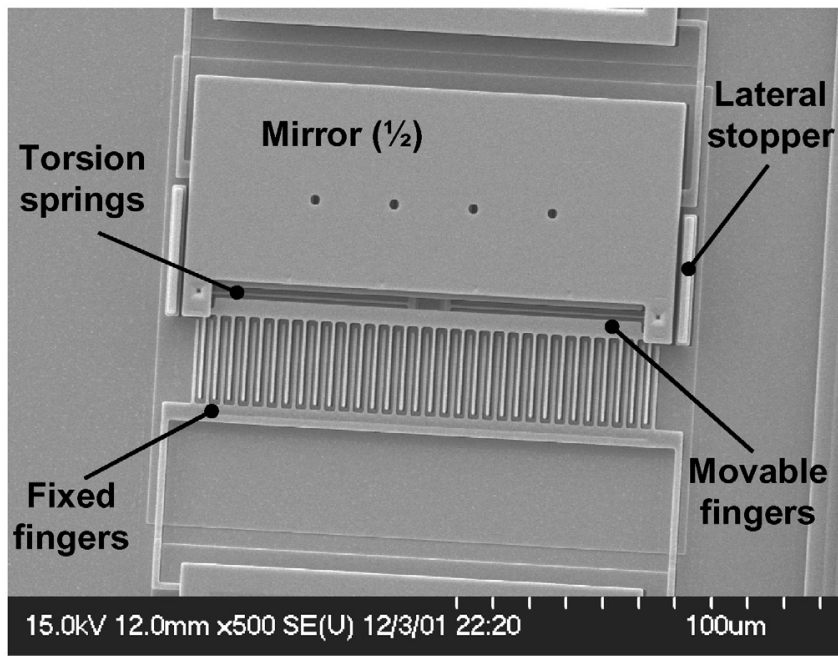

Figure 2. The SEM micrograph of the fabricated micromirror. The lower half of the micromirror was removed intentionally to reveal the underlying comb structures.

\section{THEORY}

The schematic of the analog micromirror is shown in Fig. 1. The vertical comb-drive actuators and the torsional springs were placed underneath the mirror to achieve high fill-factor, which is required for flat-top frequency response. Figure 2 shows the scanning electron microscope (SEM) image of the fabricated micromirror. Half of the mirror was intentionally removed to reveal the underlying structures. The height difference between the movable and the fixed fingers is illustrated by different contrast 
Lateral stoppers are incorporated to constrain in-plane rotation caused by lateral instability.

In contrast to parallel-plate actuators, the analysis of the vertical comb-drive actuators is more complicated due to strong fringe field, especially when there is no overlap between the comb fingers. We have developed a hybrid model that combines analytical formulation with two-dimensional finite element solutions. This model is much faster than full three-dimensional finite element method, and can be used as a design tool.

The total capacitance $\left(C_{t}\right)$ as a function of the mirror angle, $\theta$, can be calculated by integrating the sheet capacitance of a unit cell, $C_{u n i t}$, as indicated by the dotted box in Fig. 3(a):

$$
C_{t}(\theta)=2 N_{f} \cdot \int_{L_{\text {over }}} C_{\text {unit }}\left(h_{0}-y \cdot \theta\right) d y
$$

where $N_{f}, h_{0}, y$, and $L_{\text {over }}$ are the number of fingers, initial mirror height, distance from rotation axis, and overlapped finger length, respectively. $C_{\text {unit }}$ is a function of the mirror height and is computed by a 2-D finite element method (FEM) to account for the fringe field. Figure 3(b) shows the calculated distributions of electric potentials in a unit cell when a voltage $(V)$ is applied to the fixed fingers. The mirror, the movable fingers, and the shielding electrode are grounded. The mirror and the movable fingers are attracted towards fixed fingers by the electrostatic torque $\left(T_{e}\right)$ until it is balanced by a mechanical restoring torque $\left(T_{r}\right)$ :

$$
\begin{gathered}
T_{e}=\frac{V^{2}}{2} \frac{\partial C_{t}}{\partial \theta} \\
T_{r}=\frac{m G_{p o l y} W_{s} T_{s}^{3}}{3 L_{s}}\left(1-\frac{192}{\pi^{5}} \frac{T_{s}}{W_{s}} \tanh \left(\frac{\pi}{2} \frac{W_{s}}{T_{s}}\right)\right) \cdot \theta
\end{gathered}
$$

where, $m$ is number of springs with dimension of $T_{s}$ (thickness) $\times$

(a)

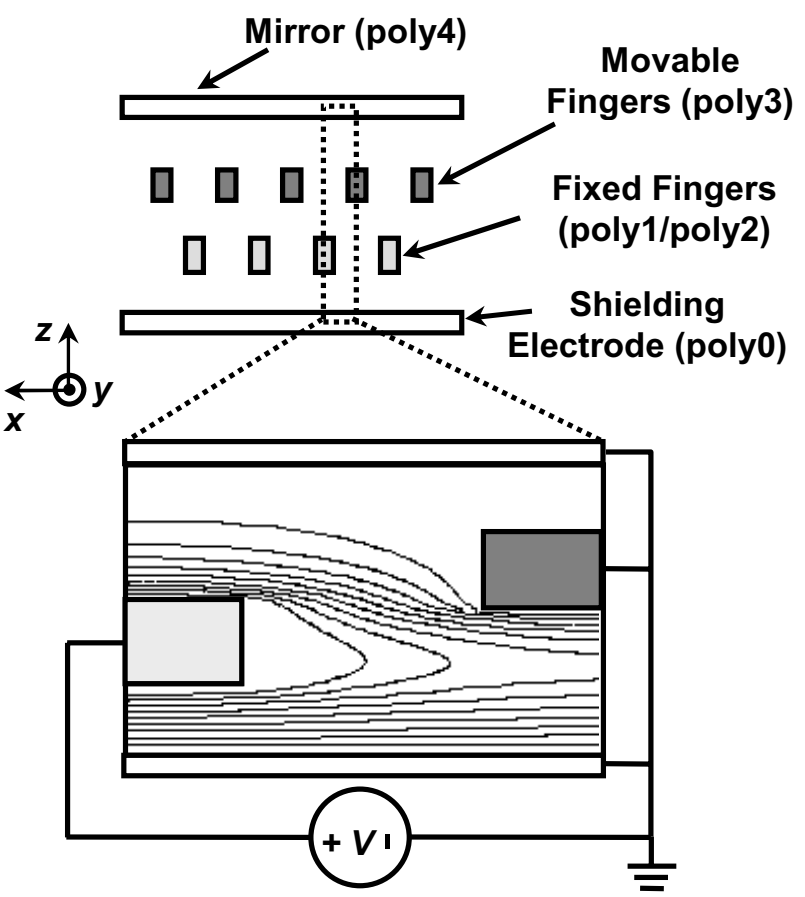

Figure 3. (a) The cross-section and (b) the calculated electric potential distribution of the proposed micromirror.
$W_{s}$ (width) $\times L_{s}$ (length), assuming $W_{s}>T_{s}$, and $G_{p o l y}$ is the shear modulus of polysilicon. At sufficiently large angle, pull-in could occur due to the parallel plate capacitances between the mirror and the top surface of the fixed electrode. Previously, we have proved that the pull-in angle $\left(\theta_{p i}\right)$ for any electrostatic actuator is only a function of the capacitance [8], and can be found by solving the following equation:

$$
\frac{\partial C_{t}}{\partial \theta}-\theta \cdot \frac{\partial^{2} C_{t}}{\partial \theta^{2}}=0
$$

\section{DESIGN}

Our design is based on the SUMMiT-V (Sandia ultra-planar multilevel MEMS technology-V) process. SUMMiT-V consists of five polysilicon layers (one is fixed and four are movable) and is ideal for implementing our devices. There are two chemicalmechanical planarization processes in SUMMiT-V: one before deposition of the poly3 layer and the other before deposition of poly4 layer. We use laminated poly1 (1 $\mu \mathrm{m}$ thick) and poly2 (1.5 $\mu \mathrm{m}$ thick) layers for fixed bottom fingers, and poly3 $(2.25 \mu \mathrm{m}$ thick) for movable top fingers. The first CMP process provides a vertical offset between the fixed and movable combs and keep the movable fingers flat and straight. The mirror is fabricated on the top polysilicon layer, poly4 (2.25 $\mu \mathrm{m}$ thick). Thanks to the second CMP process, the mirror surface is smooth and flat. A shielding electrode (poly0) is added below the fixed comb to prevent any unexpected interaction between the mirror and substrate. The cross-section of the device is shown in Fig. 3(a).

The minimum spacing between fixed and movable combs allowed in SUMMiT-V is $0.5 \mu \mathrm{m}$. The narrow gap spacing greatly increases the capacitance and therefore the force density, which can be utilized to reduce the operating voltage. The minimum finger width is $1 \mu \mathrm{m}$. The narrow finger and spacing also allow us to increase the number of fingers underneath the mirror, whose width of $137 \mu \mathrm{m}$ is fixed by the system requirement.

Our main design trade-off is reducing voltage while maintaining high enough threshold for lateral instability. It is known that narrow gap spacing in comb drive actuator can lead to lateral instability at high voltages [9]. We need to have sufficiently large safety margin to prevent lateral instability even in the presence of imperfect fabrication (e.g., misalignment between fixed and movable comb fingers). To investigate this trade-off, finger spacing ranges from 0.5 to $3 \mu \mathrm{m}$ were included in the design. The finger length (Lf) was varied from 15 to $30 \mu \mathrm{m}$. Three types of springs as shown in Fig. 4 are employed in our layout. An important criterion for the spring design is that the ratio of the spring constants for in-plane rotation (about $z$-axis), $k_{z}$, to out-ofplane rotation (about $x$-axis), $k_{x}$, should be as large as possible. From this point of view, Type $B$ spring is better than Type $C$ because the former has smaller $k_{x}$ and larger $k_{z}$. The length of the

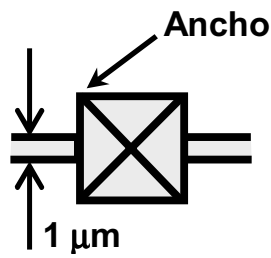

(a) Type $A$

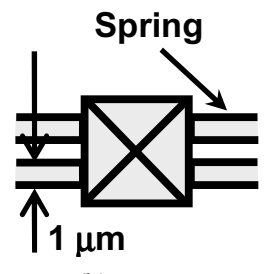

(b) Type B

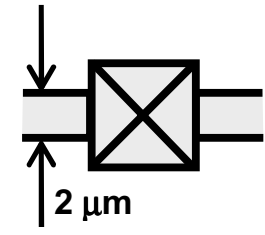

(c) Type $C$
Figure 4. Top-view schematics of the three types of torsion springs used in our design. 
spring was designed to be $55 \mu \mathrm{m}$. The mirror pitch of $150 \mu \mathrm{m}$ is determined by the system requirement. With lateral stoppers, the maximum mirror width (in $x$ direction) allowed by SUMMiT-V design rule is $137 \mu \mathrm{m}$. Therefore the linear fill-factor along the array direction is $91 \%$. The mirror length (in $y$ direction) is $120 \mu \mathrm{m}$.

\section{EXPERIMENTAL RESULTS}

The fabrication of the analog micromirror array is performed at Sandia National Lab. After SUMMiT-V process was completed, the chip was released in $\mathrm{HF}$ and dried. $\mathrm{Cr} / \mathrm{Au}$ is deposited on the whole wafer by maskless e-beam evaporation to enhance the mirror reflectivity. Electrical isolation between electrodes was achieved by incorporating overhang structures. The radius of curvature of the mirror was measured to be $135 \mathrm{~mm}$ before metallization. The stress in metal reduces that to $23 \mathrm{~mm}$ after metallization. The DC scanning characteristics of the micromirrors for various finger gap spacing, finger lengths, and torsion springs are measured by WYKO, a non-contact interferometric surface profiler. The measured results (symbols) are shown in Fig. 5, together with the calculated results (lines) obtained using our hybrid finite element method and our pull-in model [8]. The measured results agreed very well with theory. For Type A spring with $0.5 \mu \mathrm{m}$ finger spacing, a scan angle of $5.9^{\circ}$ is obtained at $6 \mathrm{~V}$ (Fig. 5(a)). The maximum angle is limited by the pull-in effect, which occurs due to the residue parallel-plate capacitances in the structure. As finger gap spacing increases (Fig. 5(a)) or finger length increases (Fig. 5(b)), the maximum angle decreases because of earlier occurrence of pull-in. In all cases, the measured pull-in angles are slightly lower than the calculated values. This is attributed to the narrow gaps $(1 \mu \mathrm{m})$ between the finger tips and the bases. The largest scan angle $\left(6.9^{\circ}\right.$ at $\left.18.1 \mathrm{~V}\right)$ is realized by short comb fingers $\left(L_{f}=15 \mu \mathrm{m}\right)$. The resonant frequencies of the micromirrors were characterized using a Polytech Microscan laser Doppler vibrometer. The resonant frequencies for various spring types are summarized in Table I. The measured results agree very well with the theoretical values. The ratio of the measured resonant frequencies is

$$
f_{R, A}: f_{R, B}: f_{R, C}=\sqrt{k_{A}}: \sqrt{k_{B}}: \sqrt{k_{C}}=1: 1.5: 2.4
$$

Fig. 6 shows the scanning electron micrographs (SEM) of the $1 \times 10$ micromirror array. The finger gap and length are $1 \mu \mathrm{m}$ and $30 \mu \mathrm{m}$, respectively. Type $A$ was used for the torsion spring. It is shown that the mirror surface remains flat in spite of the complicated topography underneath the mirror. The measured pullin angle is $6.1^{\circ}$, which is occurred at $8.8 \mathrm{~V}$. Good uniformity in the angle-versus-voltage characteristics $(< \pm 3.2 \%)$ across the array was

Table I. The measured and the calculated resonant frequencies of the micromirror.

\begin{tabular}{c|c|c}
\hline Spring type & Measured $[\mathrm{kHz}]$ & Calculated $[\mathrm{kHz}]$ \\
\hline$A$ & 3.4 & 3.5 \\
\hline$B$ & 5.1 & 5.0 \\
\hline$C$ & 8.1 & 8.0 \\
\hline
\end{tabular}

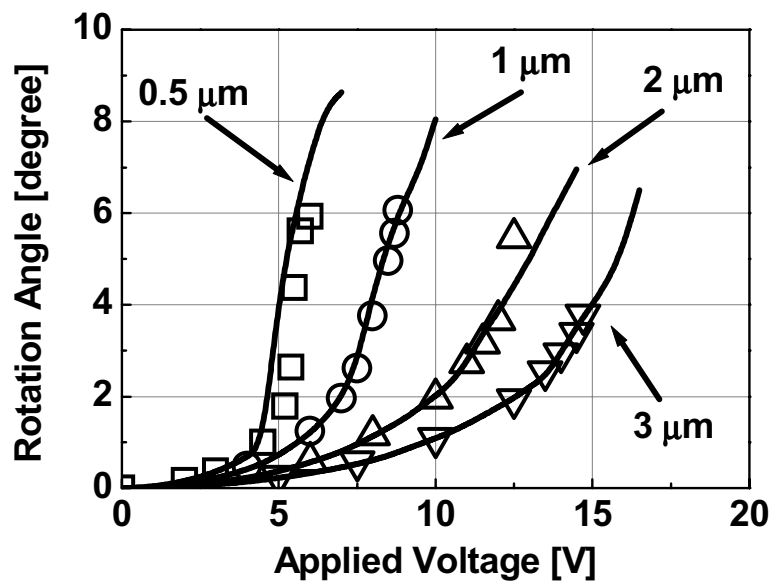

(a) For various finger gap spacing, finger length: $30 \mu \mathrm{m}$, type $\mathrm{A}$ spring.

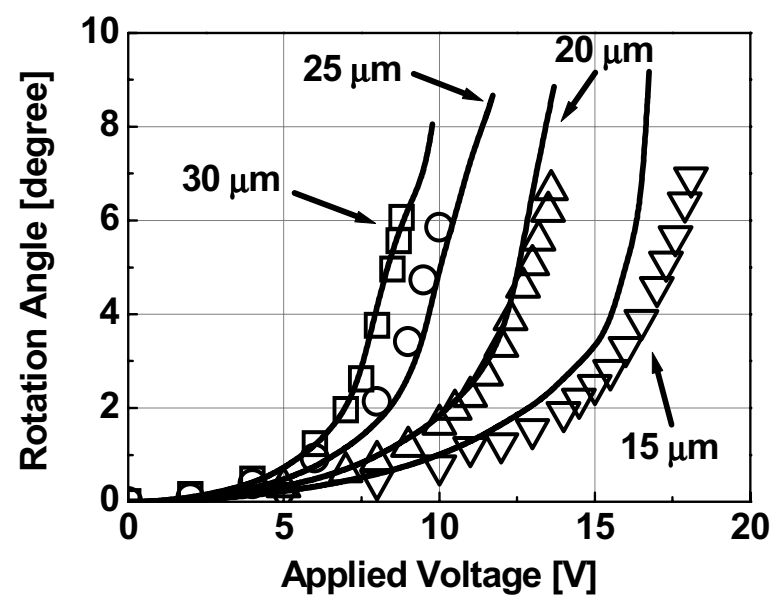

(b) For various finger length, finger gap spacing: $1 \mu$ m, type $A$ spring.

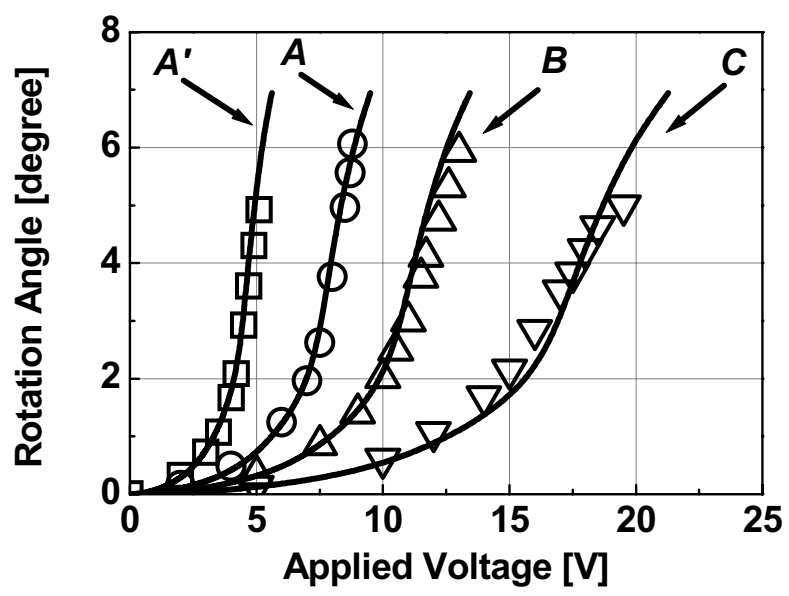

(c) For various types of springs (defined in Fig. 4), finger gap spacing: $1 \mu \mathrm{m}$, finger length: $30 \mu \mathrm{m}$. Type $A$ ' is same as type $A$ except that it is three times longer than type $A$.

Figure 5. The measured (symbols) and the calculated (lines) DC scanning characteristics of the fabricated mirrors. 


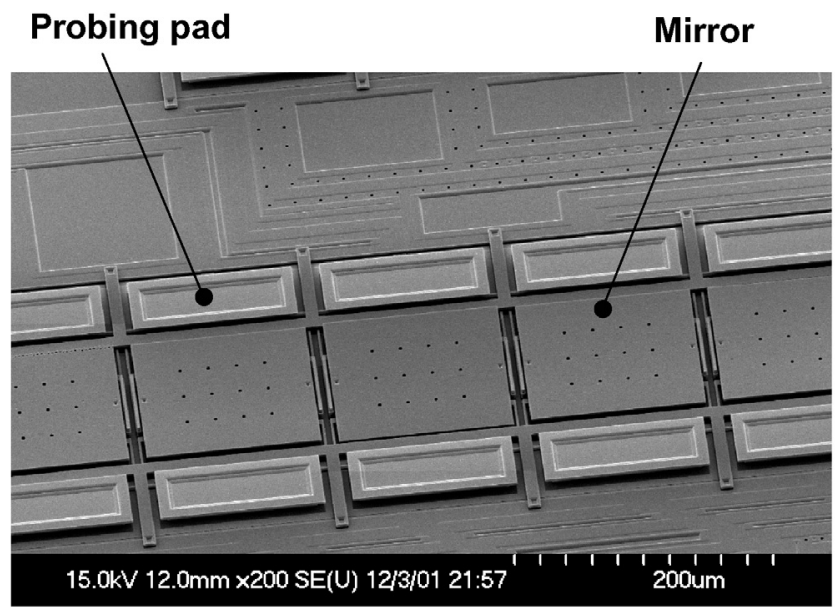

Figure 6. The SEM image of the micromirror array.

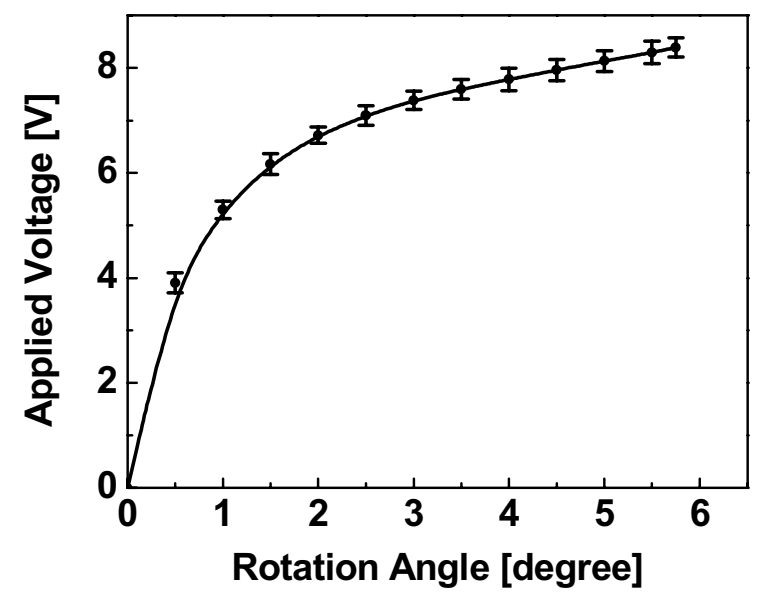

Figure 7. Uniformity of DC scanning characteristics in the $1 \times 10$ analog micromirror array.

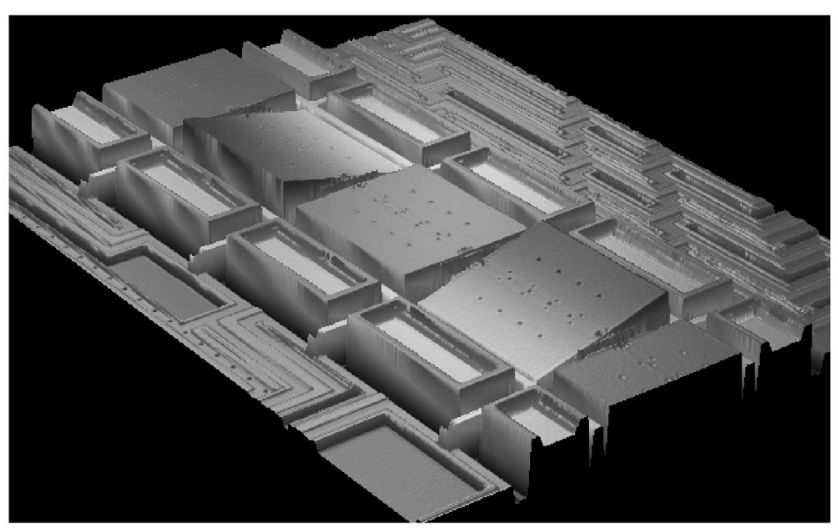

Figure 8. The WYKO image of the fabricated micromirror array. Two of the mirrors are tilted by biasing the actuators.

obtained (Fig. 7). Fig. 8 shows the optical interferometric image of the fabricated micromirror array. Two of the mirrors have been tilted by the actuators.

\section{CONCLUSIONS}

We have successfully designed and tested a 1-D analog scanning micromirror array with hidden vertical comb-drive actuators. Low operating voltage $(6 \mathrm{~V})$, wide scan range $\left(23.6^{\circ}\right.$ optical), high fill-factor (91\%), moderate resonant frequency (3.4 $\mathrm{kHz})$, and good uniformity $(< \pm 3.2 \%)$ have been demonstrated. The experimental results agree very well with our model. This micromirror array is very useful for WDM routers and WSXC.

\section{ACKNOWLEDGEMENTS}

The authors would like to thank Pamela R. Patterson, Wibool Piyawattanametha, and Erwin K. Lau of UCLA for technical assistance. This project is supported by DARPA/SPAWAR under contract N66001-00-C-8088.

\section{REFERENCES}

1. J. E. Ford, V. A. Aksyuk, David J. Bishop, and J. A. Walker, "Wavelength add-drop switching using tilting micromirrors," $J$. Light. Technol., vol. 17, pp. 904-911 (1999).

2. L. Y. Lin, E. L. Goldstein, and R. W. Tkach, "Free-space micromachined optical switches for optical networking," IEEE J. Select. Topics Quantum Electron.: Special Issue on Microoptoelectromechanical Systems (MOEMS), vol. 5, pp. 4-9 (1999).

3. A. Husain, "MEMS-based photonic switching in communications networks," Technical Digest of the OFC 2001, Anaheim, CA, 3/17-22/01, Paper WX1.

4. R. Ryf, et al., "1296-port MEMS transparent optical crossconnect with 2.07Petabit/s switch capacity," Technical Digest of the OFC 2001, Anaheim, CA, 3/17-22/01, Postdeadline Paper PD28.

5. D. Hah, S. Huang, H. Nguyen, H. Chang, H. Toshiyoshi, and M. C. Wu, "A Low Voltage, Large Scan Angle MEMS Micromirror Array with Hidden Vertical Comb-Drive Actuators for WDM Routers," 2002 Optical Fiber Communication (OFC) Conference, Anaheim, California, March 17-24, 2002

6. J.-L. A. Yeh, H. Jiang, and N. C. Tien, "Integrated polysilicon and DRIE bulk silicon micromachining for an electrostatic torsional actuator," J. Microelectromech. Syst., vol. 8, pp. 456-465 (1999).

7. R. A. Conant, J. T. Nee, K. Lau, and R. S. Muller, "A flat highfrequency scanning Micromirror," Technical Digest of the 2000 Solid-State Sensor and Actuator Workshop, Hilton Head, SC, 2000, pp. 6-9.

8. D. Hah, H. Toshiyoshi, and M. C. Wu, "Design of electrostatic actuators for MOEMS," Technical Digest of the Symposium on Design, Test, Integration and Packaging of MEMS/MOEMS 2002, Cannes-Mandelieu, France, 5/6-8/02, will be presented.

9. T. Hirano, T. Furuhata, K. J. Gabriel, and H. Fujita, "Design, fabrication, and operation of submicron gap comb-drive microactuators," J. Microelectromech. Syst., vol. 1, pp. 52-59 (1992). 